\title{
Blind Signal Classification via Sparse Coding
}

\section{Citation}

Gwon, Youngjune, Siamak Dastangoo, H. T. Kung, and Carl Fossa. 2016. "Blind Signal Classification via Sparse Coding." In Proceedings of Global Communications Conference (GLOBECOM), Washington, D.C., December 4-8, 2016.

\section{Published Version}

doi:10.1109/GLOCOM.2016.7841634

\section{Permanent link}

http://nrs.harvard.edu/urn-3:HUL.InstRepos:34390121

\section{Terms of Use}

This article was downloaded from Harvard University's DASH repository, and is made available under the terms and conditions applicable to Other Posted Material, as set forth at http:// nrs.harvard.edu/urn-3:HUL.InstRepos:dash.current.terms-of-use\#LAA

\section{Share Your Story}

The Harvard community has made this article openly available.

Please share how this access benefits you. Submit a story.

Accessibility 


\title{
Blind Signal Classification via Sparse Coding
}

\section{Youngjune Gwon, S. Dastangoo, H.T. Kung, C. Fossa}

\author{
December 5, 2016
}

The 59th IEEE Global Communications Conference, Washington, D.C.

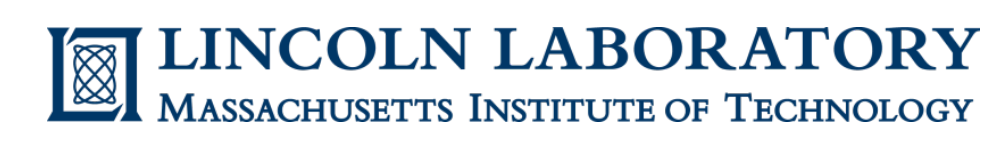

This work is sponsored by the Department of Defense under Air Force Contract FA8721-05-C-0002. Opinions, interpretations, conclusions, and recommendations are those of the author and are not necessarily endorsed by the United States Government. 


\section{Outline}

- Motivation

- Background

- Technical Approach

- Evaluation

- Results

- Summary 


\section{Motivation}

- Competing Cognitive Radio Network (CCRN) models tactical radio networks under competition

- Blue Force (friend) vs. Red Force (adversary)

- Dynamic, open spectrum resource for opportunistic data access

- Nodes are cognitive radios

$>$ Comm nodes and jammers

- Strategic jamming attacks

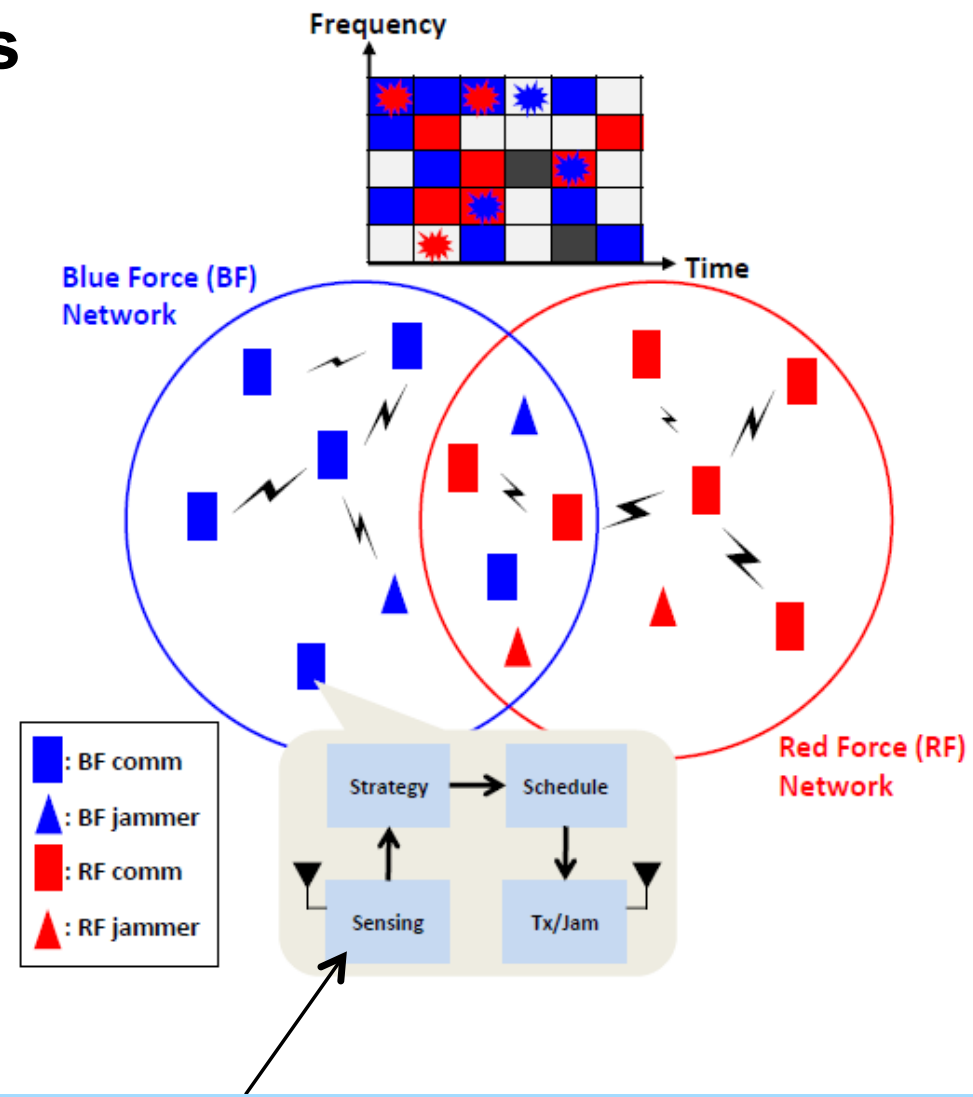

This paper is about signal classification at spectrum sensing level using semi-supervised machine learning approach 


\section{Background: Taxonomy of Spectrum Sensing}

- Non-learning based spectrum sensing

- Energy detection

- Cyclostationary detection

- Learning-based spectrum sensing

- Supervised learning (requires labeled examples of all signals you want to classify)

$>$ Support vector machine (SVM), logistic/softmax regression, neural network

- Unsupervised learning (no labeled examples required)

$>$ Clustering techniques (e.g., K-means, GMM): partition data mixed of unknown identities into clusters

- Semi-supervised (unsupervised feature learning followed by supervised phase)

$>$ Sparse coding + SVM (you need some labeled examples) 
- Sparse coding is an unsupervised learning method

- Transforms raw data into their sparse feature representations given set of basis vectors (dictionary)

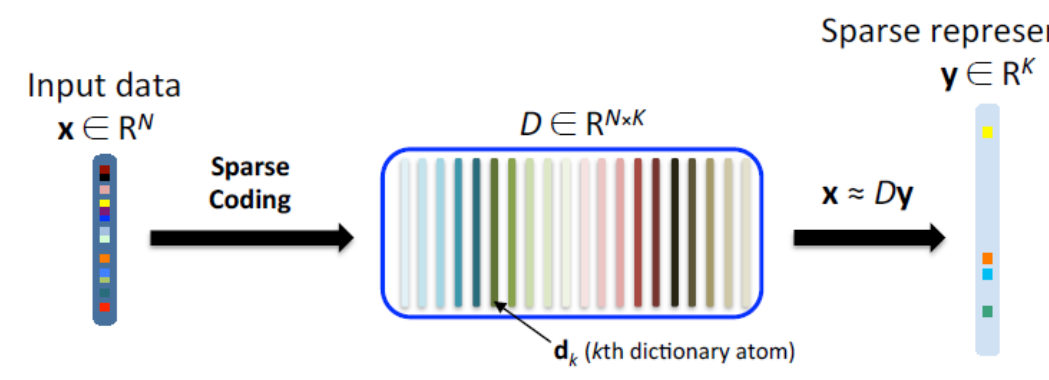

- Dictionary learnıng

- Learns basis vectors $d_{k}$ (dictionary atoms) required for sparse coding

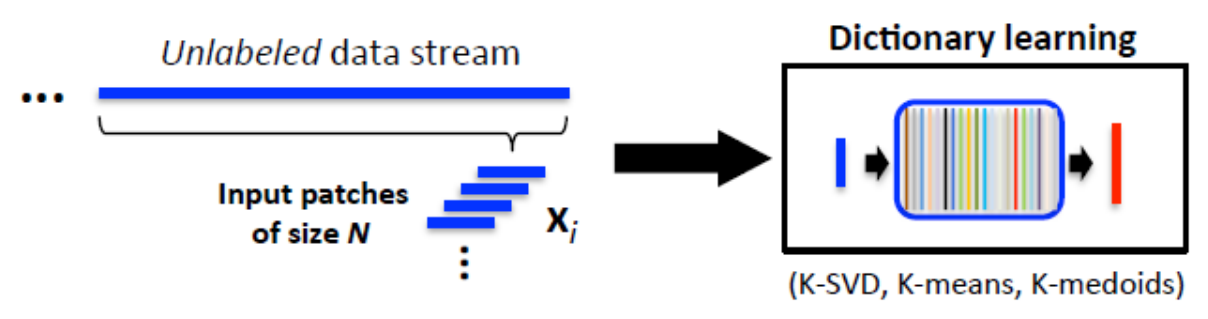




\section{Technical Approach: Semi-Supervised Learning with Sparse Coding}

- Classification pipeline

1. Extract feature vectors via sparse coding: $x_{i} \rightarrow y_{i}$

2. Summarize multiple feature vectors via pooling: $y_{i} \rightarrow z$

3. Train SVM classifiers that takes pooled sparse-coded input $z$

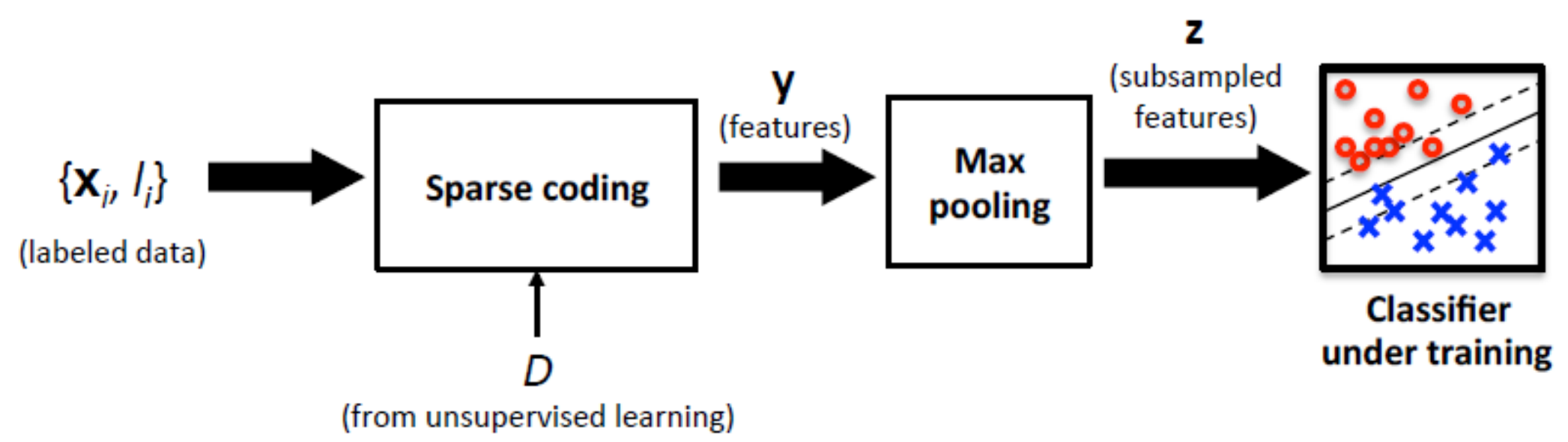

Trained SVM predicts label of unknown input data 


\section{Technical Approach (cont'd): Modification of Sparse Coder with Convolution}

- Classical inner-product sparse coders are not appropriate for our applications resulting in redundant dictionary atoms

- Received signals are time series with unknown phases

- Our enhancement: simple convolution sparse coder

- For $S$-sparse y, take $S$ steps of greedily choosing max convolution value and removing its contribution from $x$ for next

$$
\begin{gathered}
y_{i}=\max \left|\mathbf{x} * \mathbf{d}_{k}\right| \\
k \in\{1, \ldots, K\}
\end{gathered}
$$




\section{Evaluation}

- Simulation environment

- Used MATLAB communications toolbox to generate modulated RF signals

- Used LIBSVM to train SVM classifiers

- Used K-SVD algorithm to learn dictionary for sparse coding

- Assumptions

- There are four signal classes in our experiments

$>$ Friendly signals: S1 (single-carrier QPSK with rectangular pulse) and S2 (OFDM with raised cosine pulse)

$>$ Adversary signals: S3 (QPSK with custom pulse) and S4 (OFDM with custom pulse)

- Scenarios

- Case 1 (Blind clustering) - apply K-means clustering on sparse-coded signals using four classes of signals

- Case 2 (One-class SVM) - train SVM classifiers using only friendly signals

- Case 3 (1-vs-all SVM) - train SVM classifiers using mostly friendly signals and some adversary signals 


\section{Results: Confusion Matrices}

- Confusion matrix is good for visualizing multiclass classification performance

- Confusion matrices for:

- Case 1 (Blind clustering) - apply Kmeans clustering on sparse-coded signals using four classes of signals

- Case 2 (One-class SVM) - train SVM classifiers using only friendly signals

- Case 3 (1-vs-all SVM) - train SVM classifiers using mostly friendly signals and some adversary signals

Darkest box: 0.89 Lightest box: 0.06
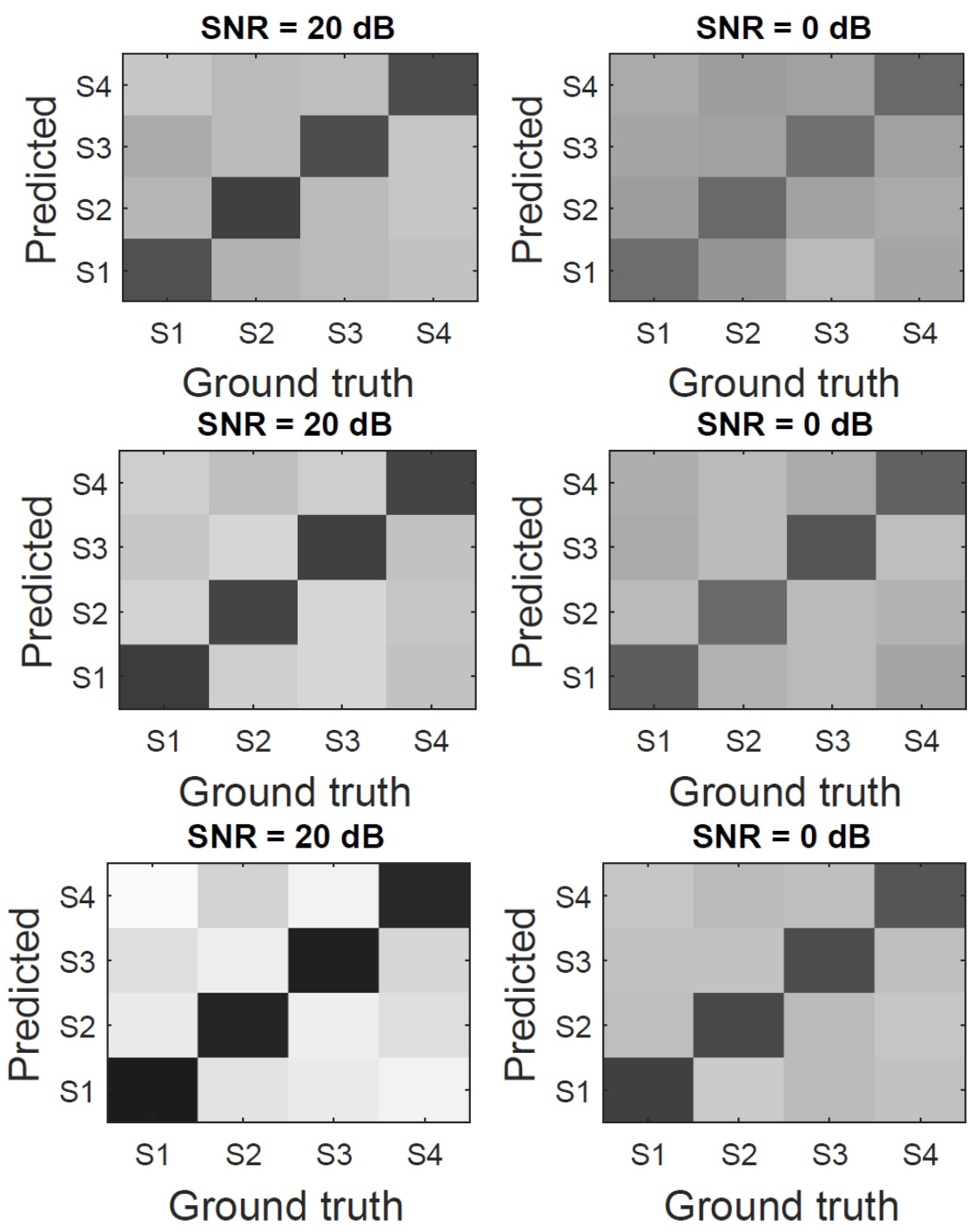


\section{Results: Recall \& False Alarm Performance}

- Recall \& false alarm performances for:

- Blind clustering - apply K-means clustering on sparse-coded signals using four classes of signals

- One-class SVM - train SVM classifiers using only friendly signals

- 1-vs-all SVM - train SVM classifiers using mostly friendly signals and some adversary signals

\begin{tabular}{|l|c|c|}
\multicolumn{1}{|c|}{ Scenarios } & $\begin{array}{c}\text { Recall } \\
20 \mathrm{~dB}(0 \mathrm{~dB})\end{array}$ & $\begin{array}{c}\text { False Alarm } \\
20 \mathrm{~dB}(0 \mathrm{~dB})\end{array}$ \\
\hline Case 1 (Blind clustering) & $0.703(0.582)$ & $0.246(0.367)$ \\
\hline Case 2 (One-class SVM) & $0.768(0.634)$ & $0.213(0.307)$ \\
\hline Case 3 (1-vs-all SVM) & $0.878(0.726)$ & $0.141(0.262)$ \\
\hline
\end{tabular}




\section{Summary}

- Presented semi-supervised framework for RF signal classification at spectrum-sensing level based on sparse coding

- Proposed sparse coding + SVM requires no prior knowledge about signals

- Sparse coding dictionary can be pre-generated or learned

- Developed simulation to assess performance for:

- Blind clustering - apply K-means clustering on sparse-coded signals using four classes of signals

- One-class SVM - train SVM classifiers using only friendly signals

- 1-vs-all SVM - train SVM classifiers using mostly friendly signals and some adversary signals

- Explore more practical applications with cognitive radios

- Improve computational complexity

- Develop efficient sparse coding and dictionary learning algorithms for mobile handsets 